\title{
Food system resilience: ontology development and impossible trinities
}

\author{
Lan van Wassenaer ${ }^{0}$, Elsje Oosterkamp, Marcel van Asseldonk and Mark Ryan*
}

\begin{abstract}
Background: Given the polysemy of the concept resilience, is it possible to have a unified conceptualisation of food system resilience? This paper provides a multidisciplinary evaluation of the literature to identify common themes that prevail in food system resilience debates and the challenges to reach a unified conceptualisation. The aim is to provide insights into some of the main issues and tensions that we identified during our research which will then allow academics and policymakers to identify the most significant themes and topics for discussion in the debate. While we only focus on one application of resilience, namely, food system resilience during extreme events, we believe that our approach and findings could be integrated and applied to other areas of resilience, as well.

Results: Our ontological exploration shows that'resilience' is a rich concept that has an important bearing on many themes and topics in the domain of food systems. This makes it highly relevant to develop and apply the concept of 'food system resilience'. The application of the concept in research and practice, however, faces many challenges that are rooted in ontological and epistemological differences among different disciplines and practices. While posing serious challenges in interdisciplinary collaboration and communication, these challenges also foster new research opportunities as knowledge gaps are discovered. We propose that there is a need to critically investigate the social, environmental, and economic trade-offs implied in policy strategies towards resilience at various levels of food systems and among different actors or groups of actors.

Conclusion: Interdisciplinary communication between different fields can be difficult and divisive. The ambition to unify the theory may be counterproductive in addressing practical problems that often require multidisciplinary collaboration. The challenge is to identify context-specific challenges and policy options using the 'resilience lens' and translating the concept into measurable indicators. Our research identifies some of these challenges and aims to provide a way to improve dialogue between different actors, through discussions about tensions and issues within their research. The ontological differences and debates are unlikely to be resolved anytime soon, nor should it be a priority to resolve these differences. A pragmatic case-specific approach can be adopted to address the problem while considering ontological differences. Our research will provide food system resilience researchers with some guidance about challenges in their research, as well as those working on developing ontologies.
\end{abstract}

Keywords: Resilience, Food systems, Ontology, Sustainability, Impossible trinities

\section{Background}

Within the resilience debate, there is often confusion and contestation about what exactly is resilience, how can we measure it, and how can varying disciplines discuss

*Correspondence: mark.ryan@wur.nl

Wageningen Economic Research, Wageningen, The Netherlands this concept and ideal in a balanced and coherent way (see e.g.,[12]. There are a lot of different terminologies, disciplinary baggage, and variant disciplines working on resilience that often talk across one another. Ecologists, social scientists, economists, and policymakers, all bring their views, interpretations, and values to the debate, and their backgrounds and understandings cause rifts in the original author(s) and the source, provide a link to the Creative Commons licence, and indicate if changes were made. The images or other third party material in this article are included in the article's Creative Commons licence, unless indicated otherwise in a credit line to the material. If material is not included in the article's Creative Commons licence and your intended use is not permitted by statutory regulation or exceeds the permitted use, you will need to obtain permission directly from the copyright holder. To view a copy of this licence, visit http://creativecommons.org/licenses/by/4.0/. The Creative Commons Public Domain Dedication waiver (http://creativeco mmons.org/publicdomain/zero/1.0/) applies to the data made available in this article, unless otherwise stated in a credit line to the data. 
discussion, thus often stifling progress. This is exacerbated in some discussions around specific types of resilience, such as food system resilience, which is often seen as an area where multiple different types of resilience align (for example, political resilience, social resilience, and agricultural resilience). This further problematises an already very challenging area of research and policy implementation.

Given the polysemy of the concept resilience, is it possible to have a unified conceptualisation of food system resilience? This paper provides a multidisciplinary evaluation of the literature to identify common themes that prevail in food system resilience debates and the challenges to reach a unified conceptualisation. The aim is to provide insights into some of the main issues and tensions that we identified during our research which will then allow academics and policymakers to identify the most significant themes and topics for discussion in the debate. While we only focus on one application of resilience, namely, food system resilience during extreme events, we believe that our approach and findings could be integrated and applied to other areas of resilience, as well.

The remainder of this paper is divided into five sections. "Objective and methodology" section outlines the objective and our methodology. The challenges in conceptualising food system resilience elaborates on the problem statement and presents findings from the literature regarding the issues, themes, topics, and concepts related to food system resilience and ontology development. ${ }^{1}$ "Results and discussion" details our findings, clearly illustrating our conceptual mapping of food system resilience, mind-maps of the most discussed concepts within the field, and our findings from ontology development. "Results and discussion" section also provides insights about several challenges that we faced in the project (the trilemmas) in developing an ontology and addressing practical problems. "Conclusion" section concludes the paper with recommendations for future work.

\section{Objective and methodology Objective}

Despite the controversies surrounding the concept of food system resilience, there is a growing demand for research to analyse and quantify the resilience of food systems in different contexts for policy objectives such as food security. Our paper is motivated by the observation that food system resilience is problematic in interdisciplinary communication and collaboration.

Our research has been led by the research question: is it possible to have a standardised description of the resilience of a system, such as a food system, with a measurable set of indicators? In our study, we seek to address this question through the development of an ontology of food system resilience, to contribute to the interdisciplinary debate. Specifically, we chose to analyse this question through the lens of food system resilience in a specific context, namely, during extreme events. Our reason for doing so is to add a distinct context and applicability for the concept of food system resilience, but also, we believe that food system resilience during extreme events is one of the most important, but also, challenging, times for ensuring food security.

The initial objective of our ontological exploration was to provide a mapping of interrelations and interactions among various concepts and classifications within the debate around food system resilience. By doing so, our project aimed to provide clear insights and the development of an ontological classification of the most used concepts within the literature on food system resilience. The preliminary goal of our ontology was to provide a guideline for interdisciplinary discussion within the field, finding a common ground through the most prevalent terms and distinctions made within the overall field. We hoped that it would provide a bridge between disciplines through a template of topics and issues that should be discussed within the debate among relevant actors. ${ }^{2}$

However, during the early stages of our project, we noticed many interesting challenges with such a goal and this paper describes some of these concerns. This paper will present the most important findings in our research to date, during our ontological development of food system resilience. The goal of the paper is not to provide this distinctive ontology, nor to present a systematic review and meta-analysis of existing literature, but rather to discuss the impossibility of developing a unified ontology. In doing so, we aim to contribute to a field fraught with different understandings of food system resilience. We are aware of the disciplinary divergences within the debate, and we believe it to be more fruitful to identify where these different definitions of food system resilience converge and diverge. Our research will move the discussion forward, by providing a common understanding of

\footnotetext{
${ }^{1}$ Ontology is a term rooted in philosophy where its meaning is the theory of existence. In the fields of artificial intelligence and knowledge management, ontology is an explicit specification of conceptualisation, i.e., formal specifications of the terms in the research domain and the relationships among them Gruber [24]
}

\footnotetext{
${ }^{2}$ While we appreciate that some of the classifications that we establish bring their own theoretical and terminological baggage, it is a first step towards understanding the array, and most important, items being discussed among the varying disciplines in resilience analysis.
} 
concepts and terminology used within the evaluation and implementation of food system resilience discourse.

\section{Methodology}

Our methodology consists of literature analysis and ontology development. The literature analysis serves both as input for and reflection on the ontology development. In general, we followed the PRIMA-protocol in selecting and documenting the literature as specified in Moher et al. [31]. It should, however, be noted that the main objective of our research was not systematic review and meta-analysis per se, but rather the development of an ontology in which literature analysis was an important component. Our presentation of the findings (in sections "The challenges in conceptualising food system resilience" and "Results and discussion") is therefore tailored to this purpose. For more standard presentation of results from systematic review, examples can be found in the work of Santeramo and Lamonaca [38].

To develop the ontology of food system resilience and extreme events, we research various guides on ontology development and used these to gather fresh insights into our ontological development (e.g., [14, 33]. We also studied academic and semi-academic literature on resilience in food systems and used insights from semantic technologies (e.g., context analysis) to derive the main topics, themes, and concepts in this domain.

The overall steps that we took in this paper were: (1) conducted a literature search using Boolean combinations, resulting in a long list of peer-reviewed articles; (2) screening for relevance; (3) content analysis and vocabulary development; and (4) ontology development. In the following paragraphs, there steps are described in more detail.

\section{Literature search}

To identify and select relevant literature, we searched the bibliographic database Scopus and the online search engine Google Scholar (search option: in the title of the article) with the following Boolean combinations: (1) Resilience AND 'food system'; (2) 'Food systems' AND 'extreme event'; (3) Ontology AND Resilience. The search was limited to studies in English that were publish between 2010 and 2020. We did not include the combination of 'resilience' and 'extreme events' because we wanted to focus on resilience in the food system and this search combination included such a wide array of other extreme events that had nothing to do with food system resilience.
The inclusion criteria for the selection of articles were:

1) Studies published between 2010 and 2020;

2) Studies published in the English language. ${ }^{3}$

\section{Screening for relevance}

The literature search has resulted in 321 peer-reviewed articles in Scopus and 52 articles from Google Scholar, of which 31 were duplicates from those found in Scopus. ${ }^{4}$ For effective content analysis and vocabulary development, the range of the literature was narrowed down to 30 peer-reviewed articles for further content analysis after being screened for relevance.

The criteria for the selection were: (1) full text (PDF) available; (2) with the main focus on the resilience of food systems.

\section{Content analysis and vocabulary development}

In further processing and analysing the literature, we combined two interrelated approaches:

1) Content analysis using text-mining software Leximancer ${ }^{5}$ and programme developed by the project team ${ }^{6}$;

2) Vocabulary development using outputs from the text-mining programmes and expert knowledge by the project team.

The software Leximancer is a text analytics tool that can be used to analyse the content of collections of textual documents and to display the extracted information visually. The software displays the information as a conceptual map that gives a bird's eye view of the main concepts contained within the text as well as information about how they are related. This is useful to explore the main themes and concepts related to resilience, food systems and extreme events. The interactive nature of the map permits the user to explore examples of concepts and their connections to each other, as well as links to the original text. This feature is particularly useful when a large amount of texts needs to be analysed.

For vocabulary development, the research team analysed the corpus of documents (scientific articles and reports) on the target domain (resilience of food systems

\footnotetext{
${ }^{3}$ The reason for this was because we wanted to focus on the concept of 'resilience,' which often did not have exact matches or accurate translations. Furthermore, we wanted to keep consistency among the literature analysed.

4 The RIS files of these references are available upon request.

5 Leximancer 5.0 https://info.leximancer.com/products-academic.

6 The program and material are available for test purposes upon request.
} 


\section{Climate Change: risk, political, social-ecological, community, dynamics, regional, role, rural adaptation, impacts, policy, services, public, capacity development, use, security, change, people, research}

Fig. 1 Climate change vocabulary example

during extreme events). The team implemented the following steps in the vocabulary development stage:

1) Applying normalised word frequency analysis to create a list of relevant words and phrases.

2) Using expert elicitation to sift through the most frequent words and phrases, select the relevant concepts from these words and phrases and add information to these concepts such as synonyms and definitions.

For these steps, we used a text-mining program, which was developed in-house. The reason for using this programme, over a third-party software, is because we have experience using it, direct access to all its functionality, and channels for quickly resolving issues if they arise, inhouse. This has resulted in a preliminary shared vocabulary about extreme events and resilience. A shared vocabulary is a list of words and phrases that are relevant to a domain together with their definitions. The vocabulary is shared in the sense that the definitions for the different concepts are agreed upon by several domainexperts (i.e. it is shared by the community). The concepts in the vocabulary are not only single words but may also be abbreviations or noun phrases ( $n$-grams) ${ }^{7}$ consisting of multiple words (e.g., 'climate change') (see Fig. 1 for one example of this).

If several words or noun phrases denote the same concept (i.e. synonyms), or are used for the same concept, they will be included in the same grouping of that concept. Words and phrases may also be ambiguous and relate to different concepts. Finally, concepts may also include translations to other languages. To facilitate the exchange of information, the vocabulary and ontology should be specified in the Resource Description Framework (RDF). This format is commonly used to specify ontologies. This will be a file containing all the concepts and their relations.

The vocabulary could be later extended with more concepts and shared with other domain experts to include relations between the different concepts. First,

\footnotetext{
${ }^{7}$ An n-gram is a term used in computational linguistics and probability to denote a contiguous sequence of ' $n$ items' of a particular sample text or speech.
}

hierarchical is-a (or type-of) relations will be added specifying parent-child relations (e.g., 'food system resilience' is a type of 'resilience'). A vocabulary with is-a relations is called taxonomy. ${ }^{8}$ Then, other types of relations will be added (e.g., 'climate change' is caused by 'carbon emissions').

\section{Ontology development}

For ontology development, we made use of the software Protégé. ${ }^{9}$ Protégé provides a graphic user interface to define and edit ontologies. The software and its documentation offer cutting-edge ways to acquire, represent, and process information [32]. Protégé ontologies can be exported into a variety of formats including $\underline{\mathrm{RDF}}$ RDFS, OWL, and XML Schema. ${ }^{10}$ There are many other ontology editors or ontology software available as well ${ }^{11}$ with similar functionalities, but we chose Protégé because it is open access; supported by a strong community of academics, government, and corporate users; and its plugin architecture, which makes it straightforward to build ontology applications, such as ours. We were able to use the architectural output of Protégé to create an intelligent system for food system resilience ontology.

\section{The challenges in conceptualising food system resilience}

In the last decade, many have emphasised the importance of improving the resilience of food systems to address societal challenges such as food security (see e.g., $[5,8$, $36,41])$. Although the attention for food system resilience seems to be a recent phenomenon, resilience has been a recurrent theme in research across various scientific disciplines and domains for many decades (see e.g., $[10,13$, $17,21,34])$. However, the concept has been difficult to define and because of the large diversity of domains using it, interdisciplinary collaboration has been strained. In

\footnotetext{
${ }^{8}$ The difference between a taxonomy and an ontology is that a taxonomy typically only refers to the hierarchical relationship between concepts, while ontology identifies different concepts and their interactions and relationships.

${ }^{9}$ Open-source software available at: https://protege.stanford.edu/.

${ }^{10}$ Explanation on these formats can be found at https://www.w3.org/2001/ sw/wiki/Protege.

${ }^{11}$ See examples at: https://www.w3.org/wiki/Ontology_editors.
} 
addition to resilience being problematic, the definition of 'food system' has also become an elusive concept. Therefore, discussions around 'food system resilience' has been even more conceptually, and practically, challenging for researchers, but also for policymakers trying to implement sustainable food security regulation. These issues have impeded food system resilience dialogue, which has been a challenge for collaboration and decision-making in food security. This section will examine a few of these issues to illustrate why it was relevant to develop a food system resilience ontology in the first place, before providing some of the results of our project.

\section{'Resilience' definitions}

Resilience is a concept that has been used in the fields of ecology, engineering, economics, psychology, philosophy, and sociology. The concept has a lot of disciplinary baggage, with different practitioners often talking over one another as they use the concept and approach in different ways. Even within disciplines, there is a lot of debate about what should, and should not, be classified as resilience. The concept has often been described as 'vague' and 'woolly' [7]. As noted by Wakefield et al. (2020; Page. 13), 'To claim that the concept of resilience lacks clarity is, by now, a well-worn refrain among critical and applied scholars alike'. Researchers from a variety of disciplines over the past two decades have detailed multiple contradictory and incompatible definitions of resilience that circulate within diverse policy and academic fields, resulting in debates about its political efficacy and pragmatic utility $[4,19,23,28,34]$.

Therefore, definitions of resilience, have so proliferated that it becomes necessary for researchers to review the definitions regularly $[1-4,6,7,11,27,29,30,43,44,46$, 47]. Generally acknowledging the appeal and elusiveness of the concept, many authors have intended to 'unpack', 'discuss', 'conceptualize,' 'clarify' or 'redefine' the concept using different perspectives (for example, 'holistic', 'integrative,' 'practical', 'ecological', and 'social') and for various purposes (for example, 'understanding,' 'measurement', and 'improving') $[15,18]$. This further adds to the diversity of definitions and expands the topics relevant to the concept.

\footnotetext{
12 System ontology refers to the different perspectives on the formation and way that we can analyse systems. For example, some ecologists view systems as predator-prey relationships, whereas others view systems as an exchange of processes, functions, and energies.

13 System boundary refers to the difficulty of establishing how to determine the parameters and bounds of a system. Because of the interrelated and mutual dependence of open systems, it is very difficult to clearly classify distinct boundaries and differentiating multiple interacting systems.
}

Olsson et al. [34] showed that the definitions of resilience often differ in the following aspects:

1) System ontology $y^{12}$

2) System boundary ${ }^{13}$;

3) The existence of multiple equilibria, thresholds, and feedback mechanisms ${ }^{14}$;

4) Self-organisation ${ }^{15}$; and

5) Function and functionalism. ${ }^{16}$

These are some of the reasons that an acceptable answer to the question 'what kind of thing is resilience' is still wanting Anderson [2]. It is very difficult to come to a unified way of discussing resilience and if it is to be useful within discussions and policy debates around food security, there needs to be a greater capacity for different disciplines and stakeholders to engage with one another on this topic. If there is no unity, or at least understandable and mutual exchange and dialogue on the topic of resilience, then it is difficult to see what purpose it serves or how it can be legitimated within policy. The very usability of the ideal of resilience comes into question.

\section{'Systems' definitions}

Despite the disagreement within the field, there is often one point of convergence among definitions of resilience: it cannot be reduced to a property of one entity alone-it can only exist in a relation (the resilience of $A$ to B) [12]. This agreement may be a good point at which to try to build a more coherent and unified discussion around system resilience. However, the understanding that resilience is a state between two entities raises another fundamental issue, namely, what is the 'system' that one is referring to. Without a clear delineation of the 'system', it is impossible to identify what an 'external factor' is for the system and what its levels of resilience are to those external factors.

As emphasised by Akkerhuis et al. [35], the concept 'system' represents an abstraction that is man-made and will be imprecise to some degree. For example, systems in nature are observed states, relationships, and dynamics between living and non-living entities within their environment. What a system is, and what it consists of, often relies on the intuition and inferences of the

\footnotetext{
${ }^{14}$ This refers to the somewhat unknowable nature of systems and what their state of equilibrium is, what happens when it is crossed, and transitions between different states.

15 Resilience theory is grounded in systems' complexity and the ability of systems to be able to self-organise and function.

${ }^{16}$ Function refers to the adaptation abilities, goal attainment, a system's ability towards integration and latency. In ecology, it refers to the ecological processes that allow the structure and ecosystem services to maintain.
} 
human observer. It is the parameters of analysis, scope, and intention of the observer that determines what constitutes 'the system'. Systems can be identified on a micro-scale (e.g., the system of an individual organ) to the macro scale (e.g., the universe as a whole). Systems can refer to ecological systems, as well as anthropogenic and human-social systems, such as the banking system, healthcare system, or political system. Therefore, trying to identify and distinguish systems is incredibly complex. This is exacerbated by systems consist of multiple subsystems, ranging from environmental, social, political, and agricultural (such as food systems).

Even when we are discussing singular systems, it is quite challenging. If we were to separate the multiple individual systems within a food system as a point of analysis, one would still need to make decisions about: (1) the volume of the system (i.e. boundaries, edge, extent); (2) what objects fall inside the volume of the system; and (3) what relationships between objects are part of the system. These are challenges that often make it difficult to operationalise system resilience in practice. Furthermore, when multiple observers are involved, reaching an identical version of a system can be an increasingly difficult and lengthy task. These problems become even more apparent when the measurements of these systems are implemented in practice, thus causing impediments to policy implementation and action.

\section{'Food system resilience' definitions}

With the challenges of defining and categorising the boundaries of resilience and systems, one is left with an even greater challenge to identify the 'food system' and finding ways to measure its resilience. For example, [41] propose that 'Food systems are social-ecological systems, formed of biophysical and social factors linked through feedback mechanisms. They comprise, at a minimum, the activities involved in food production, processing and packaging, distribution and retail, and consumption [20]' Ericksen classifies food systems as '... to be comprehensive and inclusive and to describe interactions and processes as linked, but by no means perfectly controllable or predictable' [20].

One definition of food system resilience is the 'capacity over time of a food system and its units at multiple levels, to provide sufficient, appropriate, and accessible food to all, in the face of various and even unforeseen disturbances' Tendall et al. [41]. However, even a very general definition such as this raises some stark concerns, such as what exactly is 'capacity', and what food is 'sufficient' and 'appropriate'. The authors themselves comment that 'much has still to be learned to validate food system resilience from an analytical perspective' [41].
Food systems comprise, and are dependent upon, ecological and agricultural systems for the origin of the food; but they also heavily rely on social, economic, and political systems for the distribution of food. Therefore, they are reliant on the resilience of many different interrelated and interacting systems. This further adds to the complexity of trying to define and identify food system resilience in both theory and practice. There have been several attempts to provide clarity to this very challenging area of research, but the difficulties around conceptually loaded terminology like 'resilience', 'system', and 'food system' have proven to be inhibitors towards effective discussion and progression within the field.

\section{Resilience ontology development}

There has been a few attempts to develop ontologies for resilience, which are often focused on specific types of resilience, such as supply chain and networks domains $[39,45]$. However, none have been conducted on food system resilience. One type of mapping of the resilience concept, generally understood, was done by Daniel [16]. Daniel [16] took a formal approach to develop a basic ontology of resilience that illustrates various aspects of ontology development, i.e. a glossary of terms, concepts, binary relationships, and formal axioms.

The resilience ontology as defined by Daniel [16] results in axiomatic formulations such as 'Resilience is a quality of a system and is dependent on an external factor' and 'A system has an ability which is dependent on a particular coping strategy'. Daniel's [16] conceptual map of resilience as shown in Fig. 2 is also instructive and helpful in identifying key topics that may also be related to food system resilience.

Daniel's [16] conceptual map of resilience provides a useful approach to the resilience concept and has been influential in our research on mapping food system resilience. However, it misses out on some of the important interactions and relationships in the diversity and dynamics that are often illustrated in ontological mapping. It predominantly focuses on ecological resilience interactions and does not include many of the socio-economic and political issues that related to other types of resilience, as such, those found in food system resilience (political resilience, economic resilience, and infrastructural resilience). Daniel's mapping is also only on resilience in a general sense, and many aspects within it may not necessarily be relevant for food system resilience, and likewise, there are many components of food system resilience which are too specific for Daniel's type of resilience mapping. 


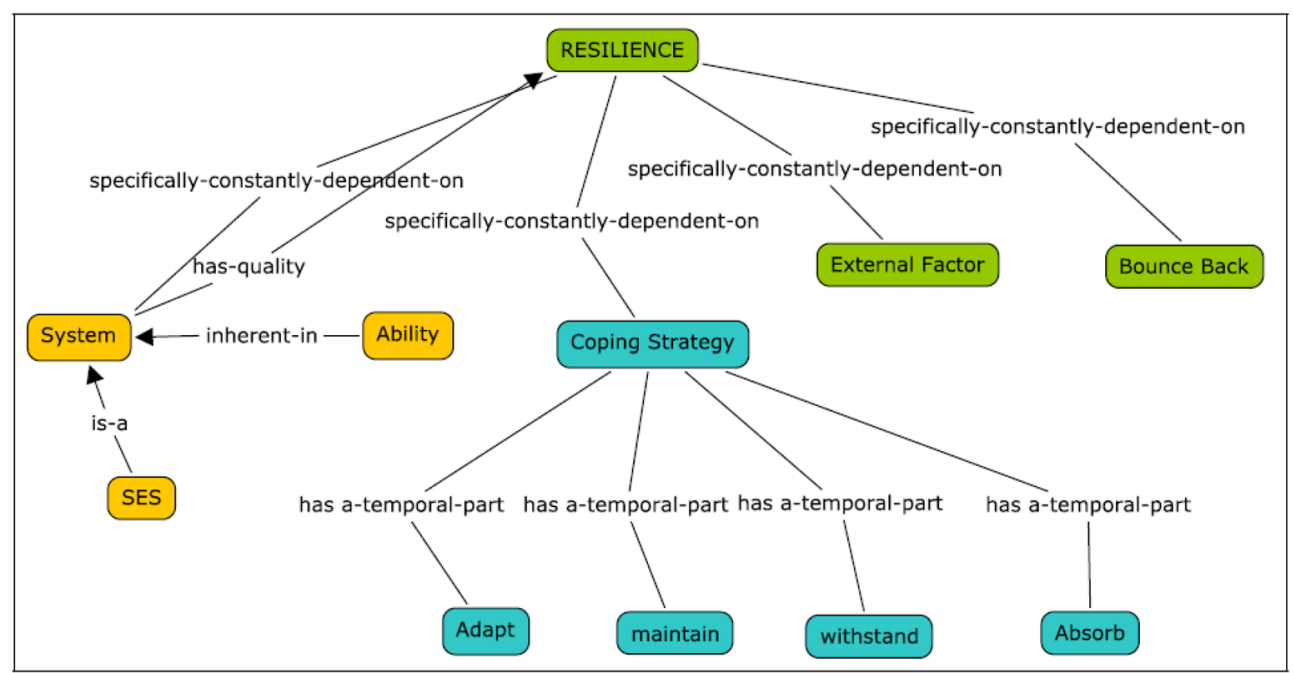

Fig. 2 A conceptual map of resilience (source: [16])

Table 1 Resilience features and concepts

\begin{tabular}{|c|c|}
\hline Resilience features & Example concept \\
\hline Resilience of what & $\begin{array}{l}\text { Agricultural systems, food systems, ecosystem, social-ecological systems, } \\
\text { information systems, networks, livestock, cities, supply chain, farmers, } \\
\text { countries }\end{array}$ \\
\hline Measuring resilience & Risk, equilibrium, indicator, capacity, capability \\
\hline Managing resilience & Risk reduction, policy, transition, strategies \\
\hline Resilience to what (disturbances) & Environmental, economic, political, technological \\
\hline Properties of resilience & Transformability, panarchy, adaptability, recovery, restoration, robustness \\
\hline Resilience for what purpose & Wellbeing, sustainability, persistence \\
\hline Lack of resilience & Insecurity, vulnerability, collapse \\
\hline Source of resilience & Redundancy, flexibility, innovation, learning, diversity, biodiversity \\
\hline
\end{tabular}

\section{Results and discussion}

\section{Findings for ontology development}

As discussed earlier coming to a unified definition of resilience is challenging. One of the reasons is the incommensurability and miscommunication in resilience definitions and measurements, which are rooted in ontological and epistemological differences between disciplines [34]. One of the methods that we suggest, and which has led to this line of research, was to understand and develop an ontology for the resilience concept. This would allow a greater understanding of the concepts being discussed within all the disciplines conducting food system resilience research, which would then bridge some of the gaps in communication and policy implementation.

A wide variety of topics are being discussed in the domain of food system resilience and these concepts were included within our shared vocabulary and used as inputs for defining the parameters of our ontology.
The first stage of our vocabulary development applied the normalised word frequency analysis to create relevant word lists that we then sifted through the most frequently used words and phrases, grouped them, and added additional synonyms and definitions. These were then grouped into categories, as illustrated in Table 1.

As already discussed, our focus is on the resilience of food systems. However, the array and diversity of topics and their intricate relationships make it challenging to find focus and priority for what would be most beneficial concepts to concentrate on for food system resilience studies. In addressing this challenge, text-mining tools such as Leximancer can be of help in identifying the areas and topics with the highest coverage in the literature, but also, the associations and linkages to other food system resilience terminology. Leximancer employs proximity values for text-mining and machine learning to automatically identify and map concepts and themes in texts [40]. In Leximancer, clusters of concepts are grouped into 


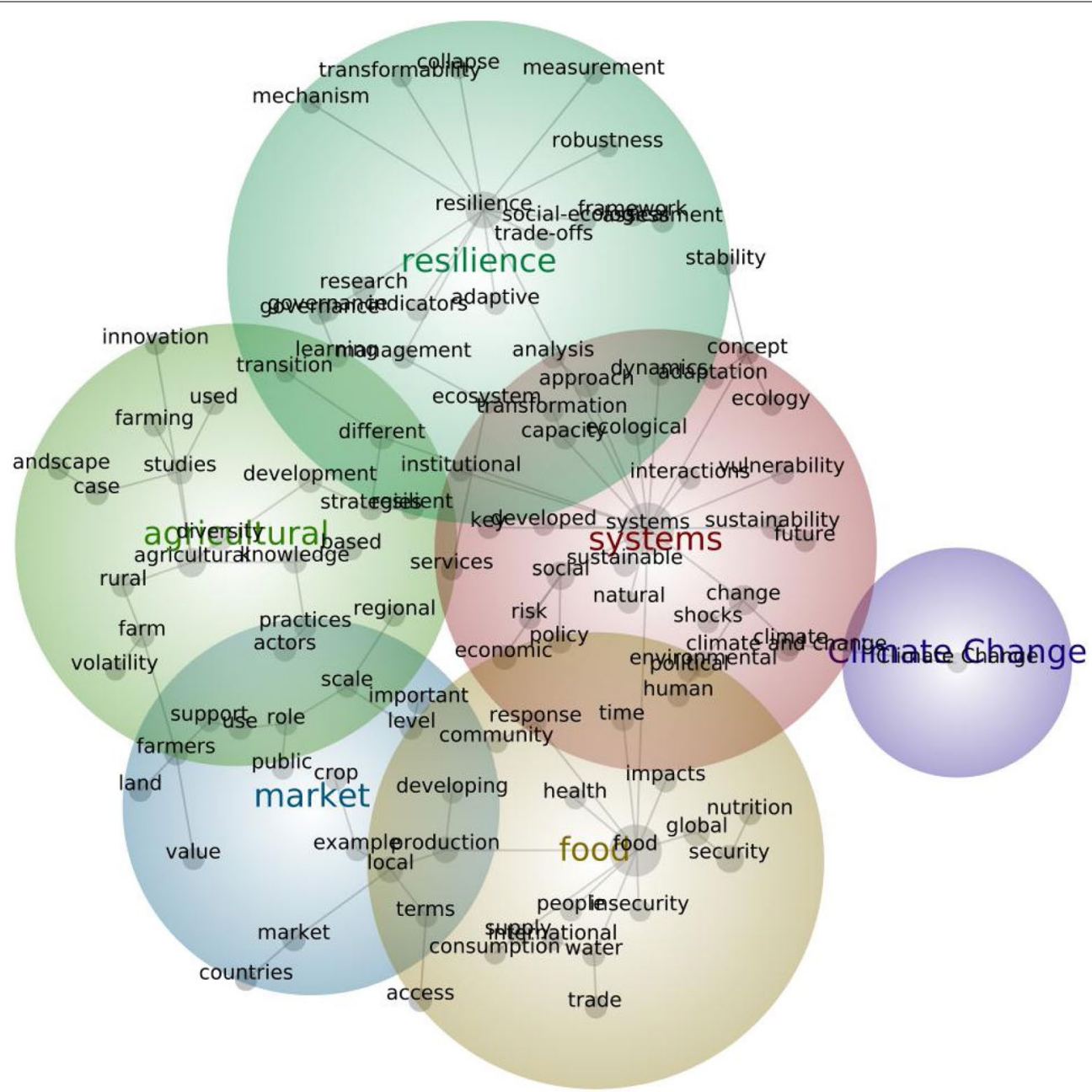

Fig. 3 Leximancer analysis results showing main themes and concepts on food system resilience

theme circles to summarise the main ideas in the cluster. The themes are named after the most prominent concept in the group, which is also indicated by the largest dot in the theme cluster. The map visually represents the strength of association between concepts and provides a conceptual overview of the semantic structure of the data. Figure 3 shows the major themes and topics that resulted from our Leximancer analysis. The comprehensive topic guide where these images were generated from can be seen in Appendix and more details available upon request from the authors.

At a first glance, the topics on food system resilience are clustered around resilience, agriculture, and systems. From this initial high-level analysis, different resilience concepts stand out as gaining the most important within the debate. Key aspects on systems (mainly food systems) change, viewed from social, economic, and environmental perspectives which reflect sustainability concerns. The three largest themes and concepts on food system resilience were agricultural, systems, and resilience, which was no large surprise, considering the terms in our search criteria and the topics that are being analysed. Each of the three refers to the conceptual map surrounding that concept: resilience (blue), systems (red), agriculture (green).

Similarly, the secondary themes and concepts were also ones that we expected to rank highly within the results of the Leximancer analysis. There were some interesting terms identified that strongly relate to the concept of resilience itself, such as 'different' and 'change'. The type of systems involved within food system resilience became clear in this analysis, with agricultural, social, and economic systems, all taking a strong role in food system resilience. There was nothing particularly shocking for the researchers in the most important themes that arose, except for 'approach' and 'studies', which probably resulted because of the largely academically oriented literature which was analysed. 


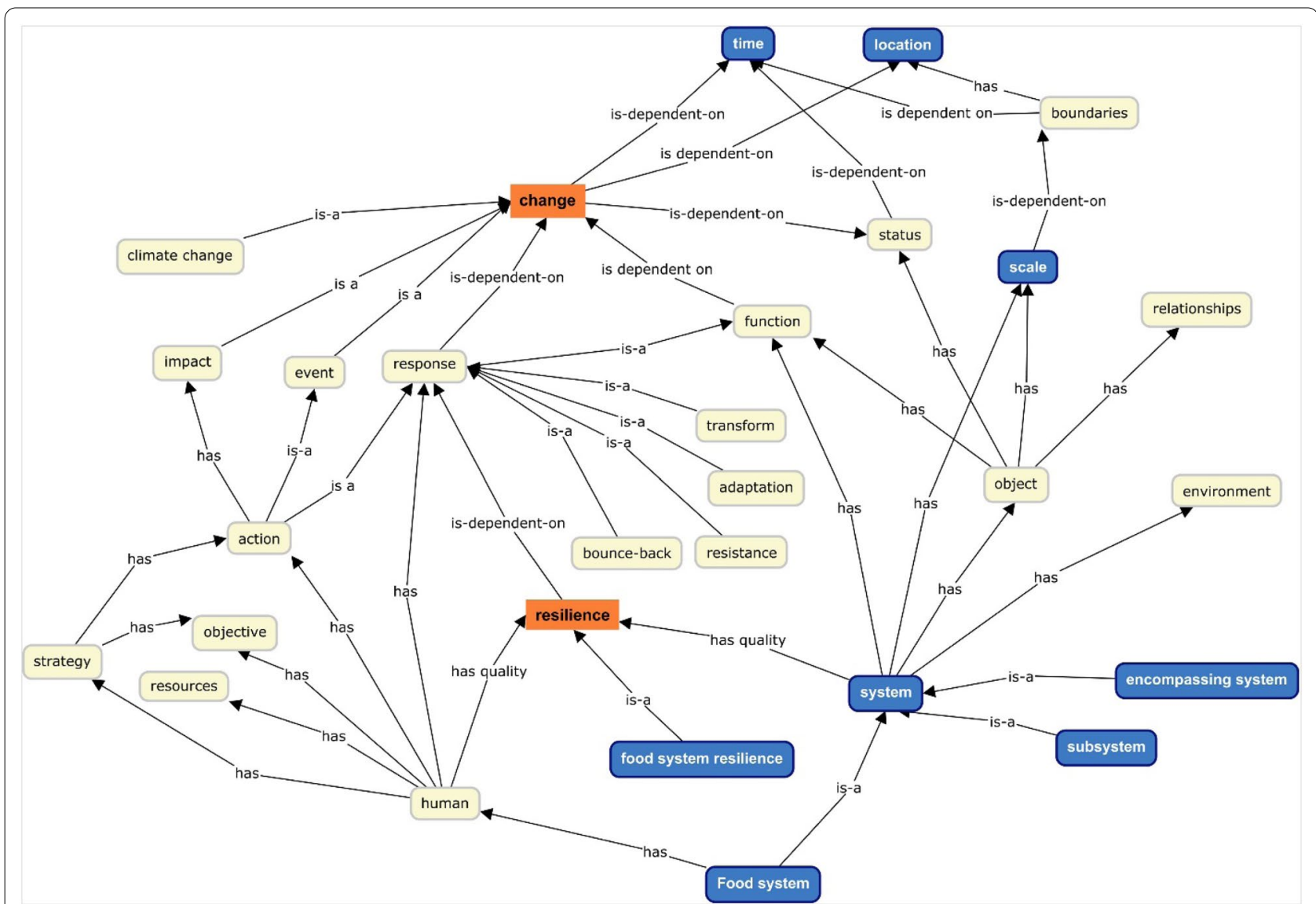

Fig. 4 Conceptual map of concepts related to food system resilience

Using the same corpus of documents, Fig. 3 provides a detailed view of the topics related to our research. This mid-level analysis gives greater insights into not only what topics are being discussed, but also, how they are related to each other. The proximity of the concepts to each of their main themes refer to how often those terms are used in connection to those terms (e.g., trade-offs is far more commonly referred in relation to resilience than say mechanism or measurement).

Based on the list of conceptual terms retrieved from our Leximancer analysis (see Appendix), and how these are visualised in Fig. 3, we have identified that the following themes emerge in our research on the resilience of food systems:

1) The impact of climate change on the sustainability of food systems (or vulnerability).

2) Trade-offs of resilience within the systems and between different features of resilience (robustness, transformability).

3) The links between food production, health, and water (impacts of the food system).
We drew these inferences from the content analysis, using Leximancer, and the proximity or size of concepts related to key clusters in this visualisation. For example, climate change has a significant emphasis and impact on theories of food system resilience, as depicted in Fig. 3. The concept of resilience is most closely aligned to issues of trade-off, but also, measurement and mechanisms were more distantly discussed. We have also aimed to capture this in our food system resilience conceptual map in Fig. 4. We use this software interface to explore the relationship between concepts in the literature and links to other known ontologies (we use this to help create our conceptual resilience map in Fig. 4).

The colours represented in Fig. 4 are to illustrate the two key concepts related to system resilience (resilience and change in orange); the most significant key concepts related to systems and aspects relevant to their characterisation (blue boxes), and the rest are lesser, but still important concepts in food system resilience.

Another step towards developing our ontology was to show the axioms relevant for its conceptualisation [16, $39,45]$. The list of axioms below is a sample size of our 
complete list, which can be found in the software that we used:

1) Resilience is dependent on the response of the system to changes;

2) Responses can be bouncing back, resistance, adapt or transform;

3) A food system is a system;

4) A system can have resilience as quality;

5) A system can have a subsystem(s) and encompassing system(s);

6) Food system resilience is a resilience (of the food system);

7) Climate change is a change;

8) Change is dependent on time;

9) Humans can have the quality of resilience;

10) Humans can have a strategy;

11) A strategy has action and serves objective(s).

These axioms are important for the structuring and implementation of food system resilience, but their deeprooted conceptualisations may also create disciplinary division. It appears through the formulation and conceptualisation of a given approach, such as food system resilience, there is the potential to constantly get trapped in further loops of conceptual division. While building an ontology does not overcome this problem, it may provide an initial step towards facilitating interdisciplinary collaboration, through a shared sense of what is held as important among the disciplines involved. While our ontological work is still in development, our research towards its development has allowed us to identify some of the key challenges involved in the construction of such an ontology in the first place.

Our ontological development will not close the debate around food system resilience, but quite the opposite, we aim to open communication channels between an array of different disciplines within an interdisciplinary reference model. Fundamentally, building ontology serves as a means, rather than an end, to facilitate interdisciplinary collaboration. It is meant as a stepping-stone, or to establish a common ground, upon which interdisciplinary collaboration can emerge. The purpose of this paper is to discuss the potential challenges involved in building a food system resilience ontology and to clarify how interdisciplinary discussions can materialise from this.

Throughout our research towards developing an ontology for food system resilience, we have identified several challenges that take the form of 'trilemmas' (also known as 'impossible trinities'), which are important to examine and understand when researching food system resilience. ${ }^{17}$ These trilemmas create theoretical tensions and methodological barriers in interdisciplinary research and provide impediments to fruitful collaborations on food system resilience. In the context of food system resilience, we identified three issues that first arose during our content analysis but kept arising throughout our research project. It is important to be aware of these three trinities of interdisciplinary food system resilience research to find dialogues and ways forward, both for research, but more importantly, for policy implementation and food security. The three aspects of food system resilience are system integrity, system transformability, and the agency of individuals (see Fig. 5).

These three aspects of food system resilience have caused great tension within the debate and impeded interdisciplinary definitions and collaborations in the area. The discussions on resilience have revealed fundamental differences in the ontology of a system and the interpretation of 'agents of change' underlying different disciplines [12]. This is demonstrated in the incommensurability among three key dimensions of food system resilience definitions:

1) The integrity or amorphic nature of the system: can the system be clearly defined with its boundaries, structures, components, and functions?

2) The type of resilience: bouncing back or transforming?

3) Whether the agency is considered in what constitutes the 'food system': does the system have a collective 'will' or is it the individuals who act on their own will and interest?

Within definitions of food system resilience, these three dimensions often cause conflict and tension between different actors. Sometimes it is possible to resolve two of the trilemmas while accepting trade-off with the third one. However, other times, there may even be an irresolvable tension between only two aspects of the trilemma. For example, this tension can be shown between the agency of individuals and system integrity. This can particularly be seen with a discussion around ecological systems and humankind's place and affordance of interaction towards them. There is a tension between the agency of individual human beings and the system's integrity. One must choose between not interfering with an ecological system, which would respect the integrity of that system to self-regulate or to interact and transform this

\footnotetext{
17 The notion of trilemmas has a long history, originating in many different areas, and refers to a trinity of objectives of which only two are possible at the same time.
} 


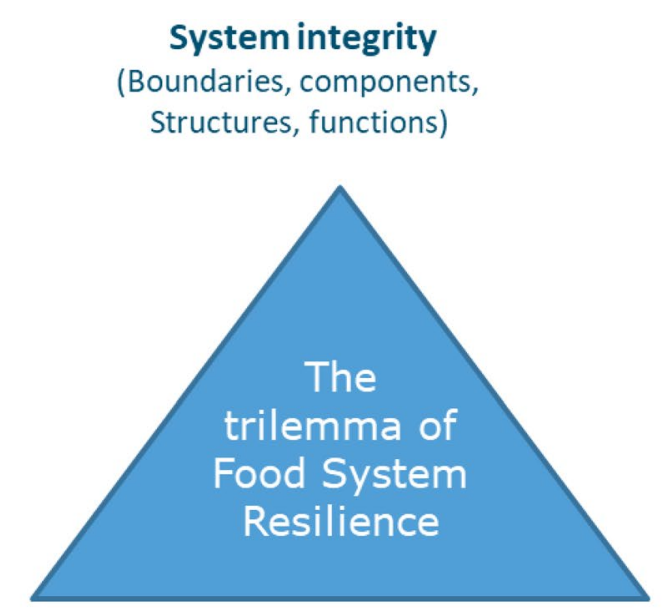

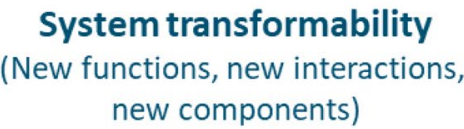

System transformability

new components)
Agency of individuals

(Rational choices, adaptation, Coordination, etc.)

Fig. 5 The trilemma in defining food system resilience

ecological system, which can be viewed as no longer respecting its integrity. This is also a common problem found within ecological literature (e.g., between compositionalists and functionalists).

Compositionalists propose that individual human action that actively changes the system is said to compromise its integrity, stating that human action is apart from, and different to, the rest of nature [9]. Whereas functionalists state that humans are just another part of nature and what is important is the overall functioning of the system, rather than its parts [37]. According to functionalists, human agency is no different from other species, so a system's integrity can no more be compromised by us than it can by other organisms and species. Both compositionalists and functionalists offer valuable perspectives for interpreting agency and integrity, but their incompatibility highlights some of the common tensions surrounding the food system resilience trilemmas, even within the same discipline, and with only two of the trilemma issues.

\section{The challenges in food system resilience research}

In developing the ontologies for food system resilience and related concepts, we have also come to realise an additional trilemma related to research on the topic of food system resilience, comprising of practical relevance, scientific rigour, and methodological feasibility (as shown in Fig. 6).

This trilemma emerged as the research team grappled with different interpretations of food system resilience and struggled to formulate a suitable modelling approach or research methods. This trilemma of having scientific rigour, methodological feasibility and practical relevance is a result of the elusiveness of concepts such as 'resilience' and 'sustainable food system' and the complexity of the 'food system. As our literature study shows, food systems are generally conceived to involve multiple objects, actors, and relationship that are embedded within multiple scales (local-national-regional-global). Changes and responses in any food system are interconnected with inherent randomness. It is therefore impossible to have all elements precisely specified and measured. However, quantitative simulations of well-defined systems with a limited set of indicators may provide insights into potential trade-offs between different objectives and system outcomes at various levels. This also concerns the development of ontologies. Terms such as 'resilience' and 'food systems' easily generate tens of thousands of articles and pose a severe constraint for research with limited time and budget constraints.

Too much focus on scientific rigour, especially from the perspective of one scientific discipline, may backfire and even result in scientific imperialism ${ }^{18}$ as suggested by Thorén and Persson [42]. However, the aspects of the trilemma do not propose that it is impossible to conduct research, but it simply points to potential issues in research that need to be identified and addressed. By firstly identifying them, it leaves us with room to integrate approaches to ensure some of these issues are

\footnotetext{
${ }^{18}$ Scientific imperialism refers to situations where science is viewed to act overbearingly. It pushes science into many, if not most, areas of human life, claiming that everything can be rationalised and calculated.
} 


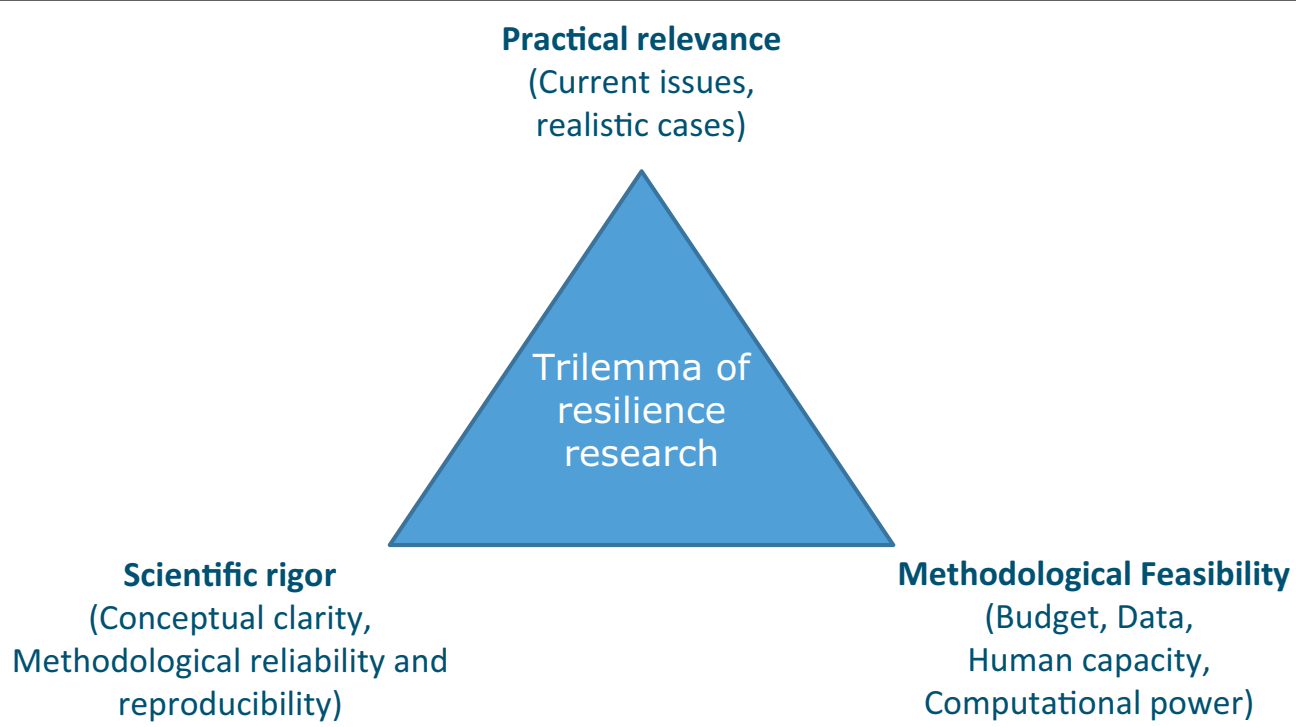

Fig. 6 The trilemma of research on food system resilience

avoided or overcome. These are important insights for researchers working in food system resilience and are worth considering when researching the area.

\section{Research opportunities and priorities on food system resilience}

Our discussion has highlighted some of the clearest challenges faced when discussing food system resilience, attempts to define it, and issues within ontological development research, more generally. The trilemmas do not mean that it is 'impossible' to study food system resilience, but rather shows the importance of being aware of the challenges within the trilemma and making explicit choices about how to address them. Our research aims to provide researchers with insights into these challenges and to assist them with interdisciplinary dialogue on the topic of food system resilience. We propose that food system resilience trilemmas may result in three innovative areas of research:

1) Research focusing on the transformability of the system and the agency of the actors, taking a transitional perspective

2) Research focusing on modelling the structure and functional relationships of the system, without considering agency issues such as power, innovation, trust

3) Research focusing on the conservation of key system functions and the agency of the actors, taking an optimisation perspective.
In our project, such discussions have resulted in the recognition of the need to better model the food system and the interactions between the resilience of individual agents (human beings with own identity, will, purpose and means) and the resilience of the food system as a collection of agents' actions (such a system has no inherent identity, will or purpose and therefore choices must be made to define the identity, will and purpose, etc.). This highlights the importance of including agency in agentbased modelling that is usually absent in current versions of it.

As observed by Carpenter et al. [10], it may seem obvious to expect that researchers should clearly state which aspect of resilience is being measured and what sorts of drivers are being considered. However, resilience in one time-period or at a particular scale can be achieved at the expense of resilience in a later period or at another scale. Understanding these transfers across scales and periods is something which should be identified in food system resilience projects.

Furthermore, methods for analysing and managing systematic risks such as 'reverse stress test' [22] and 'fault tree analysis' [26] can be used to better understand the mechanisms and pathways to systematic collapse and therefore improve resilience at various levels of the system. These modelling methods may greatly benefit the food system resilience field.

Therefore, we support some of the views within the debate that despite persistent challenges, there is a benefit to viewing food systems through a resilience lens [25]. Our exploration towards ontology development has demonstrated some of the challenges of developing 
ontologies, demonstrating that this will be an ongoing endeavour in interdisciplinary research projects, not in the least because it encourages a structured process of interdisciplinary collaboration and communication. For researchers in such research projects, ontological explorations and discussions are helpful to identify common ground and in jointly investigating knowledge gaps, critical issues, and developing novel approaches. This may lead to new insights as well as theoretical and methodological innovations. This was also evident in our research: we gained novel insights in our research about the development of ontologies, rather than necessarily the knowledge derived from the ontology itself (as it is still in development). These findings will be incorporated into the next stages of our food system resilience ontological development, but may also be beneficial to other projects working on this topic in the future.

\section{Conclusion}

While the development of our ontology is still a work in process, our research in food system resilience uncovered many valuable insights. For example, our ontological exploration shows that 'resilience' is a rich concept that has an important bearing on many themes and topics in the domain of food systems. This makes it highly relevant to develop and apply the concept of 'food system resilience.' The application of the concept in research and practice, however, faces many challenges that are rooted in ontological and epistemological differences among different disciplines and practices. While posing serious challenges in interdisciplinary collaboration and communication, these challenges also foster new research opportunities as knowledge gaps are discovered. For example, there is a need to critically investigate the social, environmental, and economic trade-offs implied in policy strategies towards resilience at various levels of food systems and among different actors or groups of actors, as demonstrated in our discussion section of the trilemma concerns.

Researching resilience in any domain is a balancing act among scientific rigour, methodological feasibility, and practical relevance. Achieving conceptual clarity as required by scientific rigour may render the research methodological infeasible due to data and budget constraints and therefore undermine the practical relevance of the research. Furthermore, the trilemmas in the domain of food system resilience now engage the core of social sciences: trade-offs, power, agency, and governance at various levels. In evaluating policy options and strategies, political ideologies and moral considerations may interfere with scientific rigour and should be made explicit in research findings. We will incorporate these insights into our research towards developing a food system resilience ontology.

Interdisciplinary communication between different fields can be difficult and divisive. The ambition to unify the theory may be counterproductive in addressing practical problems that often require multidisciplinary collaboration. The challenge is to identify context-specific challenges and policy options using the 'resilience lens' and translating the concept into measurable indicators. Our research has identified some of these challenges and aims to provide a way to improve dialogue between different actors, through discussions about tensions and issues within their research.

The ontological differences and debates are unlikely to be resolved anytime soon, nor should it be a priority to resolve these differences. If the research team aspires to address empirical problems, a pragmatic case-specific approach can be adopted to address the problem while considering ontological differences. Our research highlighted some of the key challenges while providing ways to resolve many of these concerns. It will provide food system resilience researchers with some guidance about challenges in their research, as well as those working on developing ontologies.

\section{Appendix: Topic guide and list of themes and concepts}

This appendix displays the topic guide generated by Leximancer based on a selection of peer-reviewed articles on resilience and food systems.

\begin{tabular}{lll}
\hline Word-Like & Count & Relevance (\%) \\
\hline Food & 2994 & 100 \\
Systems & 2828 & 94 \\
Resilience & 1611 & 54 \\
Agricultural & 771 & 26 \\
Change & 748 & 25 \\
Security & 612 & 20 \\
Social & 584 & 20 \\
Environmental & 495 & 17 \\
Global & 479 & 16 \\
Production & 479 & 16 \\
Local & 461 & 15 \\
Studies & 433 & 14 \\
Approach & 386 & 13 \\
Sustainability & 338 & 11 \\
Different & 337 & 11 \\
Diversity & 335 & 11 \\
Research & 322 & 11 \\
Economic & 311 & 10 \\
\hline
\end{tabular}




\begin{tabular}{|c|c|c|}
\hline Word-Like & Count & Relevance (\%) \\
\hline Sustainable & 311 & 10 \\
\hline Development & 295 & 10 \\
\hline Farm & 284 & 09 \\
\hline Farmers & 283 & 09 \\
\hline Analysis & 279 & 09 \\
\hline Access & 270 & 09 \\
\hline Market & 263 & 09 \\
\hline Vulnerability & 252 & 08 \\
\hline Capacity & 250 & 08 \\
\hline Knowledge & 249 & 08 \\
\hline Case & 244 & 08 \\
\hline Use & 242 & 08 \\
\hline Policy & 241 & 08 \\
\hline Farming & 241 & 08 \\
\hline Management & 237 & 08 \\
\hline Ecological & 237 & 08 \\
\hline Example & 233 & 08 \\
\hline Climate & 226 & 08 \\
\hline Climate and change & 226 & 08 \\
\hline Key & 222 & 07 \\
\hline Used & 220 & 07 \\
\hline Actors & 213 & 07 \\
\hline Land & 213 & 07 \\
\hline Framework & 213 & 07 \\
\hline Rural & 210 & 07 \\
\hline Water & 209 & 07 \\
\hline Supply & 207 & 07 \\
\hline Countries & 199 & 07 \\
\hline Impacts & 191 & 06 \\
\hline Level & 189 & 06 \\
\hline Important & 183 & 06 \\
\hline Trade & 179 & 06 \\
\hline Value & 177 & 06 \\
\hline Scale & 176 & 06 \\
\hline Assessment & 175 & 06 \\
\hline Resilient & 174 & 06 \\
\hline Time & 171 & 06 \\
\hline Role & 168 & 06 \\
\hline Regional & 167 & 06 \\
\hline Concept & 166 & 06 \\
\hline Indicators & 165 & 06 \\
\hline Governance & 164 & 05 \\
\hline Governance & 164 & 05 \\
\hline Political & 163 & 05 \\
\hline Adaptive & 163 & 05 \\
\hline People & 163 & 05 \\
\hline Based & 162 & 05 \\
\hline Strategies & 151 & 05 \\
\hline Human & 151 & 05 \\
\hline Terms & 149 & 05 \\
\hline Natural & 147 & 05 \\
\hline
\end{tabular}

\begin{tabular}{|c|c|c|}
\hline Word-Like & Count & Relevance (\%) \\
\hline Learning & 146 & 05 \\
\hline Future & 146 & 05 \\
\hline Ecosystem & 144 & 05 \\
\hline Innovation & 143 & 05 \\
\hline Practices & 131 & 04 \\
\hline Support & 131 & 04 \\
\hline Nutrition & 128 & 04 \\
\hline Consumption & 123 & 04 \\
\hline Dynamics & 122 & 04 \\
\hline Social-ecological & 122 & 04 \\
\hline Services & 120 & 04 \\
\hline Response & 119 & 04 \\
\hline Community & 118 & 04 \\
\hline Crop & 118 & 04 \\
\hline Public & 118 & 04 \\
\hline Interactions & 117 & 04 \\
\hline Health & 111 & 04 \\
\hline Risk & 111 & 04 \\
\hline Shocks & 110 & 04 \\
\hline Adaptation & 108 & 04 \\
\hline Landscape & 106 & 04 \\
\hline Ecology & 105 & 04 \\
\hline Insecurity & 104 & 03 \\
\hline International & 101 & 03 \\
\hline Stability & 96 & 03 \\
\hline Transformation & 84 & 03 \\
\hline Institutional & 79 & 03 \\
\hline Robustness & 74 & 02 \\
\hline Developing & 71 & 02 \\
\hline Developed & 57 & 02 \\
\hline Measurement & 51 & 02 \\
\hline Trade-offs & 48 & 02 \\
\hline Transition & 44 & 01 \\
\hline Volatility & 42 & 01 \\
\hline Mechanism & 38 & 01 \\
\hline Transformability & 37 & 01 \\
\hline Collapse & 14 & 00 \\
\hline
\end{tabular}

Exported from Leximancer output

\section{Acknowledgements}

Not applicable.

\section{Authors' contributions}

The authors read and approved the final manuscript.

\section{Funding}

Wageningen University \& Research Funding: KB-35-005-001.

\section{Availability of data and materials}

Not applicable. 


\section{Declarations}

Ethics approval and consent to participate

Not applicable.

\section{Consent for publication}

Yes.

\section{Competing interests}

The authors declare that they have no competing interests.

Received: 6 February 2021 Accepted: 12 August 2021

Published online: 29 September 2021

\section{References}

1. Adger WN, Brown K, Waters J. Resilience. Oxford: The Oxford Handbook of Climate Change and Society; 2012.

2. Anderson B. What kind of thing is resilience? Politics. 2015;35(1):60-6.

3. Bahadur AV, Ibrahim M, Tanner T. Characterising resilience: unpacking the concept for tackling climate change and development. Climate Dev. 2013:5(1):55-65.

4. Béné C. Resilience of local food systems and links to food securitya review of some important concepts in the context of COVID19 and other shocks. Food Secur. 2020. https://doi.org/10.1007/ s12571-020-01076-1.

5. Béné C, Oosterveer P, Lamotte L, Brouwer ID, de Haan S, Prager SD, Talsma EF, Khoury CK. When food systems meet sustainability-Current narratives and implications for actions. World Dev. 2019;113:116-30.

6. Bhamra R, Dani S, Burnard K. Resilience: the concept, a literature review and future directions. Int J Prod Res. 2011;49(18):5375-93.

7. Brand FS, Jax K. Focusing the meaning ( $\mathrm{s}$ ) of resilience: resilience as a descriptive concept and a boundary object. Ecol Soci. 2007:12(1):23.

8. Bullock JM, Dhanjal-Adams KL, Milne A, Oliver TH, Todman LC, Whitmore $A P$, Pywell RF. Resilience and food security: rethinking an ecological concept. J Ecol. 2017;105(4):880-4.

9. Callicott JB. Beyond the land ethic: more essays in environmental philosophy. Albany: Suny Press; 1999.

10. Carpenter S, Walker B, Anderies JM, Abel N. From metaphor to measurement: resilience of what to what? Ecosystems. 2001;4(8):765-81.

11. Chandler D. Beyond neoliberalism: resilience, the new art of governing complexity. Resilience. 2014;2(1):47-63.

12. Chandler D, Grove K, Wakefield S. Resilience in the anthropocene: governance and politics at the end of the world. Milton Park: Routledge; 2020.

13. Cote M, Nightingale AJ. Resilience thinking meets social theory: situating social change in socio-ecological systems (SES) research. Prog Hum Geogr. 2012;36(4):475-89.

14. Cristani M, Cuel R. A comprehensive guideline for building a domain ontology from scratch. In: proceeding of International Conference on Knowledge Management (IKNOW' 04). Graz, Austria. 2004.

15. Dakos V, Carpenter SR, van Nes EH, Scheffer M. Resilience indicators: prospects and limitations for early warnings of regime shifts. Philos Trans Royal Soc B Biol Sci. 2015;370(1659):20130263.

16. Daniel DC. A formal theory of resilience. Germany: University of Munster; 2011.

17. Darnhofer I. Resilience and why it matters for farm management. Eur Rev Agric Econ. 2014;41(3):461-84

18. Doorn N. Resilience indicators: opportunities for including distributive justice concerns in disaster management. J Risk Res. 2017;20(6):711-31.

19. El Chami D, Daccache A, El Moujabber M. How could sustainable agriculture increase climate resilience? A systematic review. Sustainability. 2020;12(8):3119.

20. Ericksen PJ. What is the vulnerability of a food system to global environmental change? Ecol Soc. 2008;13(2):14.

21. Folke C, Carpenter SR, Walker B, Scheffer M, Chapin T, Rockström J. Resilience thinking: integrating resilience, adaptability and transformability. Ecol Soc. 2010;15(4):20.

22. Grigat D, Caccioli F. Reverse stress testing interbank networks. Sci Rep. 2017:7(1):1-11.
23. Grove K. Resilience. Milton Park: Routledge; 2018.

24. Gruber TR. A translation approach to portable ontology specifications. Knowl Acquis. 1993;5(2):199-220.

25. Hodbod J, Eakin H. Adapting a social-ecological resilience framework for food systems. J Environ Stud Sci. 2015:5(3):474-84

26. Kishita Y, McLellan BC, Giurco D, Aoki K, Yoshizawa G, Handoh IC. Designing backcasting scenarios for resilient energy futures. Technol Forecast Soc Chang. 2017;124:114-25.

27. Klein RJ, Nicholls RJ, Thomalla F. Resilience to natural hazards: how useful is this concept? Glob Environ Change Part B Environ Hazards. 2003:5(1):35-45.

28. Li T, Dong Y, Liu Z. A review of social-ecological system resilience: mechanism, assessment and management. Sci Total Environ. 2020;723:138113.

29. Marston JM. Modeling resilience and sustainability in ancient agricultural systems. J Ethnobiol. 2015;35(3):585-605.

30. Maruyama H. Taxonomy and General Strategies for Resilience. In: Yamagata Y, Maruyama H, editors. Urban Resilience: Advanced Sciences and Technologies for Security Applications.Cham:Springer; 2016. https://doi. org/10.1007/978-3-319-39812-9_1.

31. Moher D, Shamseer L, Clarke M, Ghersi D, Liberati A, Petticrew M, Shekelle $P$, Stewart LA. Preferred reporting items for systematic review and metaanalysis protocols (PRISMA-P) 2015 statement. Syst Rev. 2015;4(1):1-9.

32. Musen MA. 'The Protégé project: a look back and a look forward. Al Matters. 2015. https://doi.org/10.1145/2557001.25757003'

33. Noy NF, McGuinness DL. Ontology development 101: A guide to creating your first ontology, Stanford knowledge systems laboratory technical report KSL-01-05. 2001. https://protegewiki.stanford.edu/wiki/Ontol ogy 101.

34. Olsson $L$, Jerneck $A$, Thoren $H$, Persson J, O'Byrne D. Why resilience is unappealing to social science: Theoretical and empirical investigations of the scientific use of resilience. Sci Adv. 2015;1(4):e1400217.

35. op Akkerhuis GAJ. Evolution and transitions in complexity: the science of hierarchical organization in nature. Berlin: Springer; 2016.

36. Pingali $P$, Alinovi L, Sutton J. Food security in complex emergencies: enhancing food system resilience. Disasters. 2005;29:S5-24

37. Ryan M. Human value, environmental ethics and sustainability: the precautionary ecosystem health principle. Lanham: Rowman \& Littlefield: 2016.

38. Santeramo FG, Lamonaca E. Evaluation of geographical label in consumers' decision-making process: a systematic review and meta-analysis. Food Res Int. 2020;131:108995.

39. Singh S, Ghosh S, Jayaram J, Tiwari MK. Enhancing supply chain resilience using ontology-based decision support system. Int J Comput Integr Manuf. 2019:32(7):642-57.

40. Smith $A E$, Humphreys MS. Evaluation of unsupervised semantic mapping of natural language with Leximancer concept mapping. Behav Res Methods. 2006;38(2):262-79.

41. Tendall D, Joerin J, Kopainsky B, Edwards P, Shreck A, Le Q, Krütli P, Grant $M$, Six J. Food system resilience: defining the concept. Glob Food Sec. 2015;6:17-23

42. Thorén H, Persson J. The philosophy of interdisciplinarity: sustainability science and problem-feeding. J Gen Philos Sci. 2013;44(2):337-55.

43. Thorén H, Persson J. Resilience: some philosophical remarks on ostensively and stipulatively defined concepts. Sustain Sci Pract Policy. 2015;11(1):64-74.

44. Urruty N, Tailliez-Lefebvre D, Huyghe C. Stability, robustness, vulnerability and resilience of agricultural systems. A review. Agron Sustain Dev. 2016;36(1):1-15.

45. Vlacheas PT, Stavroulaki V, Demestichas P, Cadzowm S, Gorniak S, Ikonomou D. Ontology and taxonomies of resilience. ENISA report. 2011.

46. Walker J, Cooper M. Genealogies of resilience: from systems ecology to the political economy of crisis adaptation. Secur Dialogue. 2011;42:143-60.

47. Xu L, Marinova D. Resilience thinking: a bibliometric analysis of socioecological research. Scientometrics. 2013;96(3):911-27.

\section{Publisher's Note}

Springer Nature remains neutral with regard to jurisdictional claims in published maps and institutional affiliations. 